\title{
Comparative Study of Different Indirect Laser-Based Methods Developed for Microprocessing of Transparent Materials
}

\author{
Béla HOPP*, Tomi SMAUSZ, Tamás CSIZMADIA, Csaba VASS, Tamás CSÁKÓ and Gábor SZABÓ \\ ${ }^{*}$ Hungarian Academy of Sciences and University of Szeged, Research Group on Laser Physics, H- \\ 6720 Szeged, Dóm tér 9, Hungary \\ E-mail: bhopp@physx.u-szeged.hu \\ Department of Optics and Quantum Electronics, University of Szeged, H-6720 Szeged, Dóm tér 9, Hungary
}

\begin{abstract}
Micro-optics and micro-fluidics are dynamically developing research areas. However, the preparation of micro-optical elements, channels and structures is a rather complicated process. The indirect laser etching methods, i.e. the backside wet and dry etching techniques (LIBWE, LIBDE) and the plasma assisted ablation (LIPAA) developed for micromachining of transparent materials are promising techniques. In this paper our aim was to accomplish a reliable comparison of these procedures. We applied same absorber material, tin in liquid and solid states, for wet-, dry- and plasma assisted etchings, respectively. The metal-fused silica interfaces were irradiated by a $\mathrm{KrF}$ excimer laser beam, the fluence was varied between 350 and $2000 \mathrm{~mJ} / \mathrm{cm}^{2}$. Measuring the depth of the etched holes we found that LIBWE method had the highest etch rate, while the etch rates of LIBDE and LIPAA were similar in the investigated fluence range. On the basis of the etch rate-fluence graphs we estimated the threshold values which were 340,360 and $500 \mathrm{~mJ} / \mathrm{cm}^{2}$ for wet-, dry- and plasma assisted etchings, respectively. EDX analysis of the etched hole bottoms demonstrated that significant number of tin atoms were built into the surface layer of the fused silica developing a mixture layer which can play an important role in the etching processes.
\end{abstract}

DOI: $10.2961 /$ jlmn.2010.01.0017

Keywords: Micromachining, transparent materials, tin, LIBWE, LIPAA, LIBDE

\section{Introduction}

Micro-optics and micro-fluidics are dynamically developing research areas. However, the preparation of different micro-optical elements, channels and structures is a rather complicated process. The indirect laser etching methods developed for micromachining of transparent materials are promising techniques. The most important members of this group are the laser induced backside wet and dry etching techniques (LIBWE, LIBDE) and the laser induced plasma assisted ablation (LIPAA).

In LIBWE, a liquid with high absorption capacity at the applied laser wavelength contacting the backside of the transparent material is irradiated by laser pulses through the solid sample. The pulse energy is absorbed and transformed into heat in a thin liquid layer contacting the transparent target. This hot liquid film warms up the surface layer of the solid sample by heat diffusion and this heated volume becomes soft or melts depending on the reached temperature. Meantime, bubbles form in the irradiated absorbing liquid layer, expand and hit against the softened or molten surface of the target inducing material damage and ejection [1-3]. Upon the use of organic absorbing solutions photochemical reactions develop and consequent dissociation of the molecules results in deposition of carbonic layer on the laser irradiated area thus promoting the further etching [4]. The most frequently applied lasers and liquid absorbers in the LIBWE procedure were the nanosecond excimer lasers (KrF, ArF, XeF; FWHM= 20-30 ns) and pyrene/toluene [5], pyrene/acetone [6], different halogenated $\left(\mathrm{C}_{6} \mathrm{H}_{5} \mathrm{~F}, \mathrm{C}_{6} \mathrm{H}_{5} \mathrm{Cl}, \mathrm{C}_{6} \mathrm{H}_{4} \mathrm{Cl}_{2}\right)$ [7] and naphthalene/methyl-methacrylate solutions [8], respectively.
Zimmer et al. used liquid metals, gallium [9] and mercury [10] as absorbers, too. The most studied transparent material was fused silica [11].

The investigations proved that the wet etching method is quite effective, however, its application is somewhat complicated in practice. This technique requires a liquid chamber with good insulation and a liquid absorber which is often a harmful solution. For elimination of these problems we developed a modified method where the absorbing liquid of LIBWE was replaced by a solid thin film. By the analogy of LIBWE this method was called laser induced backside dry etching (LIBDE). In this procedure the pulse energy is completely absorbed in the absorbing layer contacting the transparent material surface and heats up the thin film and the contacting target material. At high enough fluence the absorbing film is completely boiled while the upper layer of the transparent material can also be boiled or melted. The vapor cloud derived from the boiling absorbing material leaves the target and the induced recoil forces attack its plasticized surface resulting in the ejection of a certain part of the boiled/melted transparent material. The investigated absorbing film materials were metals: e.g. silver [12] copper, aluminium [13], tin [14]; oxides: for example $\mathrm{SiO}$ [15]; and carbon [16].

Laser induced plasma assisted ablation (LIPAA) is quite similar to the LIBDE procedure. In the LIPAA method a bulk metal target is placed behind the transparent sample with a distance up to a few tens of micrometers. The etching is induced by the energetic species of the plasma plume generated by laser ablation at the irradiated metal surface [17-19]. 
All three methods have already proved their suitability for microprocessing of transparent materials and preparation of micro-optical elements [20-26]. It is an intriguing and complex question to determine the efficacy of these methods. The aim of this study was to develop a reliable method to compare the above-mentioned indirect laser etching techniques. We realized that this is a rather difficult problem, as the generally applied solid and liquid absorbers have very different optical and thermal properties significantly influencing the machining processes. In our comparative investigations we applied the same absorber material, tin in liquid and solid states (as thin film or bulk target), for wet-, dry- and plasma assisted etchings of fused silica.

\section{Experiments and results}

\subsection{Etch rate investigation}

In our LIBWE investigations a small amount molten tin was spread on the surface of the $1 \mathrm{~mm}$ thick fused silica plate and kept in liquid state using a hot air gun. The temperature and the thickness of the molten Sn were approx. $300{ }^{\circ} \mathrm{C}$ and $1-2 \mathrm{~mm}$, respectively. In the LIBDE method the target plate was coated by a $86 \mathrm{~nm}$ thick tin film, while in the LIPAA experiments a $2 \mathrm{~mm}$ thick tin plate was directly placed onto the transparent sample. All the experiments were carried out in air. The beam of a $\mathrm{KrF}$ excimer laser was focused onto the tin-fused silica interface in a round shaped $0.72 \mathrm{~mm}^{2}$ area spot with a $10 \mathrm{~cm}$ focal length quartz lens (Fig. 1).

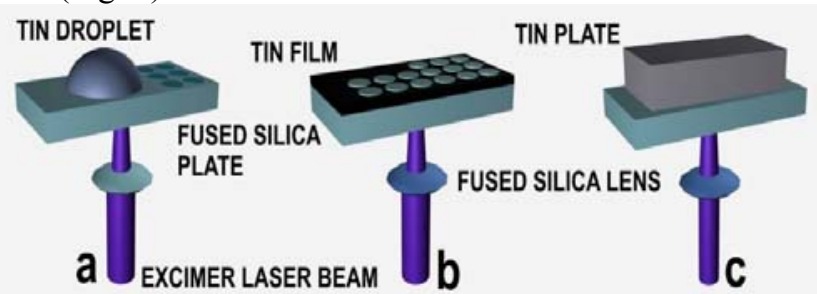

Fig. 1 Experimental arrangements for comparative investigations of LIBWE, LIBDE and LIPAA procedures

The laser fluence was adjusted by an attenuator and measured shot by shot. Accordingly, the fluence varied between 350 and $2000 \mathrm{~mJ} / \mathrm{cm}^{2}$. The applied pulse numbers were 50, 1 and 100 in the cases of LIBWE, LIBDE and LIPAA, respectively, to reach accurately measurable etch depths on the target plate surfaces. The sample fused silica plates were moved by an $x-y-z$ translator after laser etching. After irradiation the residual tin was removed chemically using nitric acid. The etch depth was measured by a DEKTAK 8 surface profiler. Figure 2. shows the characteristic cross-sections of the etched holes at different fluences.

The maximum depth of a given etched hole was divided by the applied number of laser pulses to calculate the characteristic etch rate values for all the investigated methods. Figure 3. shows the etch rates in the function of the laser fluence.

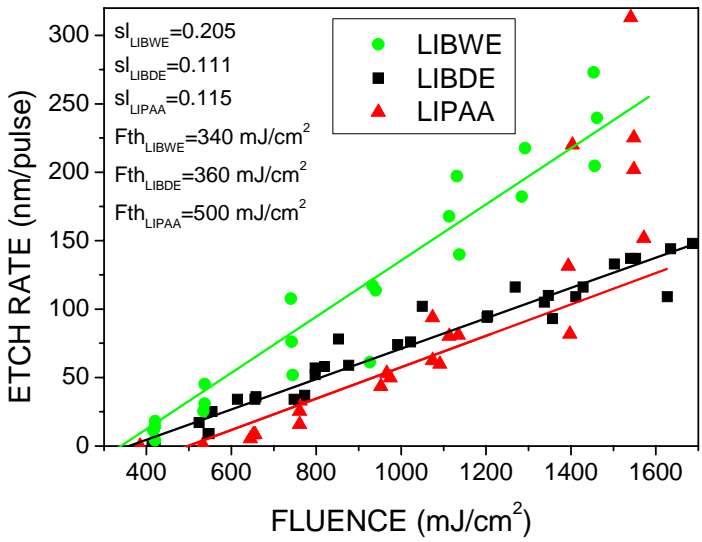

Fig. 3 Etch rates of the different procedures as a function of the applied laser fluence. The etching threshold values were determined on the basis of the lines fitted to the measured data.

It can be seen that the etch rate-fluence functions are nearly linear for each procedure in the investigated range. In the case of LIPAA this statement is valid below 1300 $\mathrm{mJ} / \mathrm{cm}^{2}$, while above this value a steep increase can be observed. It was found that the LIBWE method had the highest etch rate, while there was no significant difference between the etch rates of LIBDE and LIPAA. The etch threshold fluences estimated on the basis of the plots were 340,360 and $500 \mathrm{~mJ} / \mathrm{cm}^{2}$ for wet-, dry- and plasma assisted etchings, respectively.

The dependence of the etched depth on the number of laser pulses was also investigated. It was found that this was linear for LIBWE and LIPAA and exponential decaylike for LIBDE (Fig.4).

These are in good agreement with the earlier results obtained for other absorbing materials in the cases of LIBWE $[1,6,9]$ and LIBDE [14], too.
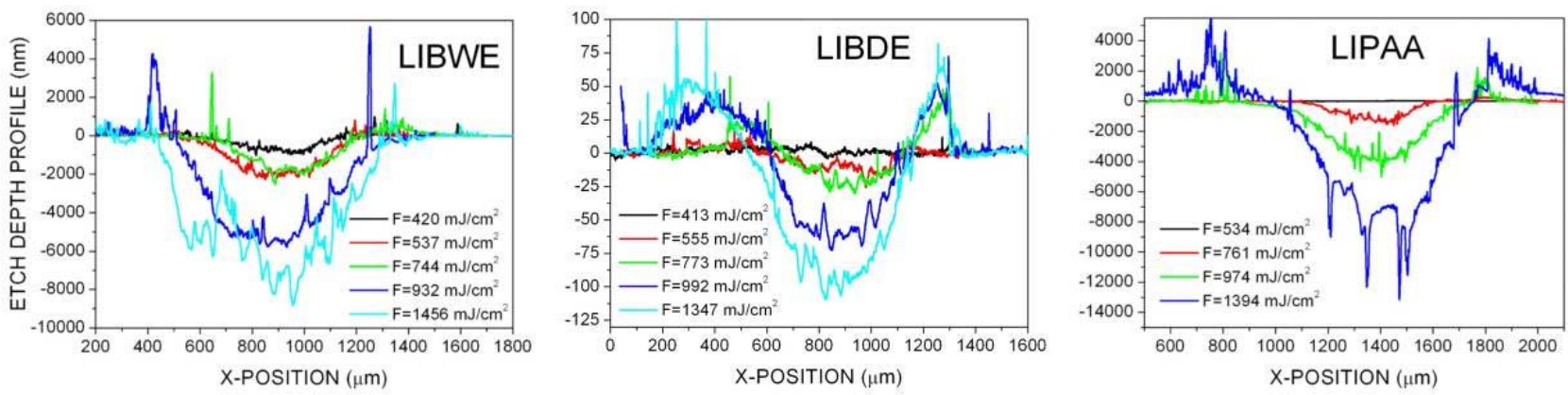

Fig. 2 Characteristic cross-sections of the etched holes at different laser fluences for the investigated methods recorded by a surface profiler. 

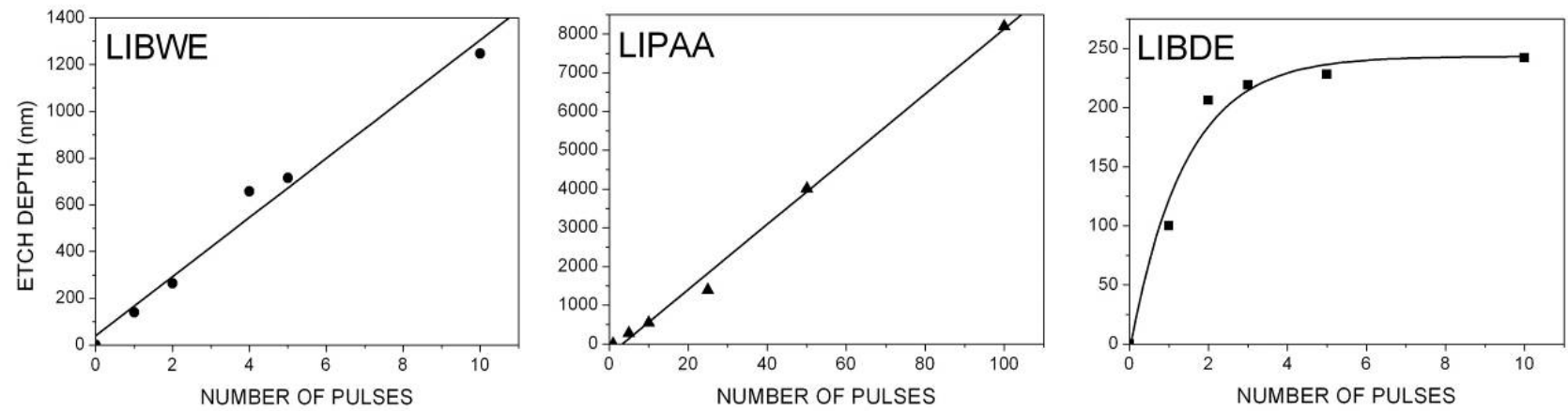

Fig. 4 The dependence of the etched depth on the number of laser pulses in the cases of the studied micromachining techniques. The applied laser fluence was $1160 \mathrm{~mJ} / \mathrm{cm}^{2}$.

\subsection{Morphological studies}

On the basis of the cross-section curves we assumed that the considerable change in the tendency of the etch rate-fluence function for LIPAA can be due to the very rough surface of the etched holes produced by the highfluence irradiation. Therefore the etched holes were investigated by a Hitachi S4700 scanning electron microscope (SEM). Figure 5. shows the SEM pictures about the bottoms of etched holes using different fluences.

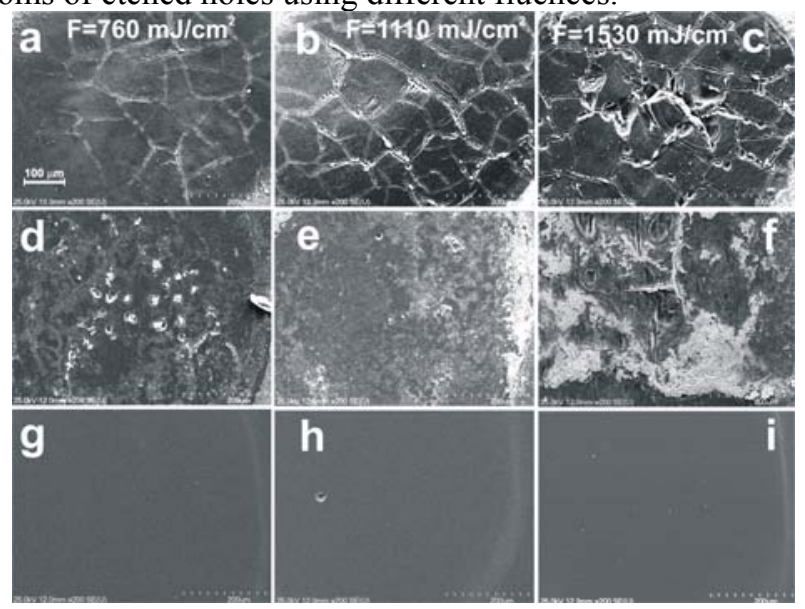

Fig. 5 Scanning electron microscopic pictures about the bottoms of the produced holes for LIPAA $(a, b, c), \operatorname{LIBWE}(\mathrm{d}, \mathrm{e}, \mathrm{f})$ and $\operatorname{LIBDE}(\mathrm{g}, \mathrm{h}, \mathrm{i})$ at different laser fluences. The actual depths of the studied holes can be determined on the basis of Fig.3.

It can be seen clearly that the etched surfaces are cracked during LIPAA, and by increasing the applied laser fluence the developed cracks become much rougher. Figure 5.c suggests that splinters were broken out from the fused silica surface. Significant distortions of the surface can be seen in the case of LIBWE (Fig.5.f). According to our assumption the rather rough surface structure can be due to the adhesion of developed acid resistant tin-oxide islands where the necessary oxygen atoms may derived from the surrounding air and the decomposed $\mathrm{SiO}_{2}$ molecules. In contrast to these the etched surfaces produced by the LIBDE procedure were found very smooth (Fig.5.i).

The Ra roughness parameter values determined on the basis of the profilometric measurements for the different methods and different fluences (Fig.6.a) showed significant differences. In the case of wet etching Ra showed a proportional growth with the in-creasing fluence. Similar, but more pronounced increase was seen for the plasma assisted ablation, while in the case of the dry etching Ra remained approximately constant. Since the obtained etch depths strongly differed for the studied methods such a direct comparison of the Ra parameters can not really compare the quality of the holes. However the reach of similar well-measurable etched depths for all of the three procedures was not realizable in consequence of the strongly limited etch depth of LIBDE. Other possibility was to investigate etched holes having small depth produced by only a few laser pulses for LIBWE and LIPAA, however in this case the measured depths showed very high deviation (low reproducibility). Therefore a relevant comparison could be the Ra roughness parameters divided by the hole depths as the function of laser fluence (Fig.6.b). In this case no significant differences could be observed for the three methods.
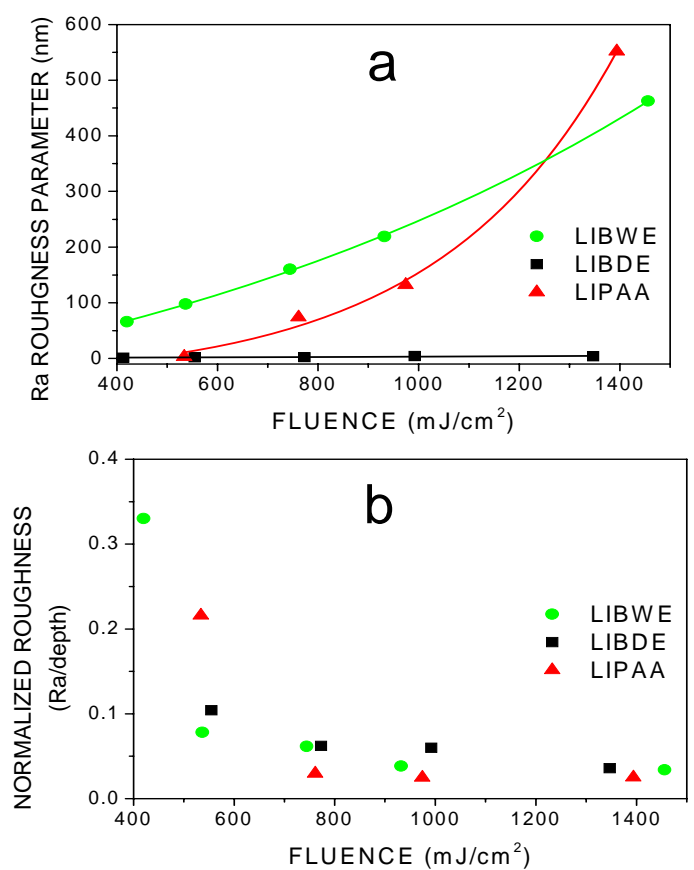

Fig. 6 Ra roughness parameters (a) and their values divided by the hole depths (b) in the function of laser fluence for the investigated methods

\subsection{EDX measurements}

The energy dispersive X-ray analysis (EDX) was applied to determine the Sn content of the surface layer of the etched hole bottoms after the nitric acid bathing. Table 1 . 
Table 1 Results of the EDX spectroscopic measurements. The applied fluences were approx. $1530 \mathrm{~mJ} / \mathrm{cm}^{2}$, the number of pulses were 50, 100 and 1 for LIBWE, LIPAA and LIBDE, respectively.

\begin{tabular}{lcccc}
\hline & Au content [at\%] & Pd content [at\%] & Sn content [at\%] & Estimated Sn layer thickness [nm] \\
\hline LIBWE & 1.01 & 0.19 & 0.56 & 29 \\
LIPAA & 0.98 & 0.39 & 0.29 & 17 \\
LIBDE & 1.1 & 0.21 & 0.08 & 4 \\
\hline
\end{tabular}

shows these values for the investigated methods. The applied fluences were around $1530 \mathrm{~mJ} / \mathrm{cm}^{2}$, the number of pulses were 50, 100 and 1 for LIBWE, LIPAA and LIBDE, respectively.

It can be seen clearly that considerable amount of tin is mixed/diffused into the fused silica during the material processing, resulting in the increase of absorption coefficient in the actually uppermost layer of the target area. This mixed layer can influence the etching mechanism and efficiency for the subsequent pulses. In the EDX analysis the geometry of the sampling volume is nearly raindrop-like having approx. 1 micrometer penetration depth depending on the investigated material [27]. We assumed that the "neck" of the drop was nearly cylindrical. Its diameter corresponds to that of the e-beam, and its length is approximately 50-100 nm. This cylinder includes the upper layer of the investigated sample and the $45 \mathrm{~nm}$ thick goldpalladium coating (Fig.7.).

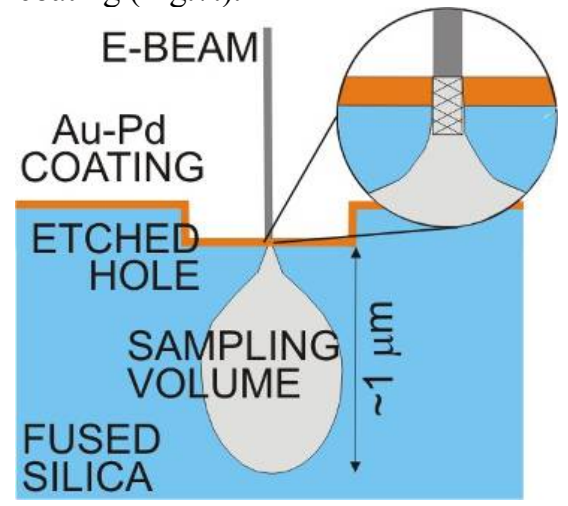

Fig. 7 Rough sketch of the supposed sampling volume of the EDX analysis in the etched fused silica sample.

We know the spatial position and total amount of the gold and palladium in the studied volume. We assumed that the majority of the detected tin atoms are also found in the cylinder. On the basis of these data and suggested conditions we could estimate the number of the tin atoms and consequently the thickness of the layer formed by them. This value is an underestimation because the metal atoms are mixed with the $\mathrm{SiO}_{2}$ molecules in the uppermost layer of the etched hole bottoms. Nevertheless these calculations can prove that the amount of the incorporated tin atoms is significant and therefore can influence the etching mechanism and main parameters in the cases of the studied methods.

\subsection{Thermal calculations to estimate the thickness of the molten layer}

The thickness of the molten fused silica layer produced during the different etching processes was calculated. We believe that this gives a reliable estimation for the maximum etch rate value, because according to our assumption part of the softened, molten fused silica volume is removed during the etching process. Our calculations are based on the absorption of pulse energy and the thermal processes (heat diffusion and phase transitions) taking place during/following the laser irradiation. All the three investigated procedures were modeled with the one-dimensional heat flow equation solved using the finite difference method.

The relevant layers of both fused silica and tin were divided into several thin membranes each having $2.5 \mathrm{~nm}$ thickness, while the time step resolution was $0.1 \mathrm{ps}$. The temporal shape of the applied excimer laser pulse was approximated by the Gaussian intensity profile, with pulse duration of $30 \mathrm{~ns}$ (FWHM). In our calculations we considered the phase changes and the temperature dependence of the necessary thermal parameters, while working with constant (temperature-independent) absorption coefficient of tin $\left(4.05 \times 10^{5} \mathrm{~cm}^{-1}\right)$. The initial temperatures of the modeled systems were $300{ }^{\circ} \mathrm{C}$ for LIBWE, and $20^{\circ} \mathrm{C}$ for LIBDE and LIPAA. In the cases of wet etching and plasma assisted ablation the considered thicknesses of tin and fused silica layers were around $10 \mu \mathrm{m}$ (the temperature rise was negligible far from the tin - fused silica boundary), while in LIBDE the tin film was $86 \mathrm{~nm}$ thick.

Our model can not take the direct material removal (the evaporation of thin tin film) into consideration during the dry etching process, therefore the obtained results are valid
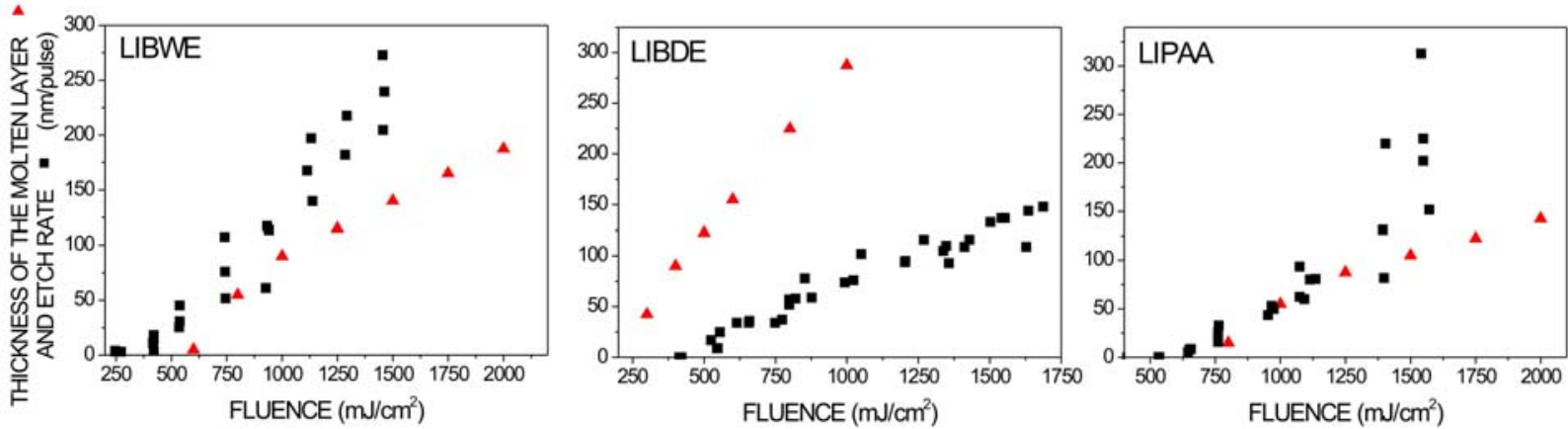

Fig. 8 Calculated thicknesses of the molten fused silica layers and the measured etch rates for LIBWE, LIBDE and LIPAA. 
only up to $400 \mathrm{~mJ} / \mathrm{cm}^{2}$ fluence rate. Above this fluence value our model is only a rough estimation (the complete boiling of the absorbing metal layer starts at around this value, but in the calculation the boiled tin remains in contact with the fused silica). This validity limit does not exist in the cases of LIBWE (the glass-metal boundary layer is closed - the tin can not be removed from the fused silica surface). The validity of our model is also limited in the case of LIPAA. It did not take into account the plasma generation and thermal connection is assumed to occur between the fused silica and tin plate. The latter one is the critical problem since a gap is developed between the etched fused silica sample and the ablated tin plate after a certain number of pulses. Fig. 8 shows the calculated thicknesses of the molten fused silica layer and the etch rates in the function of the applied laser fluence for the studied methods. It can be seen that the calculated and measured values are in good agreement.

\section{Summary and conclusions}

During our investigations we compared three indirect laser-based etching methods (LIBWE, LIBDE and LIPAA), developed for laser micromachining of transparent materials, under similar experimental conditions. The comparative study was made possible by the application of tin as absorbing material in liquid and solid (thin film and bulk) phases.

It was found that the LIBWE method had the highest etch rate, while the etch rates of LIBDE and LIPAA were quite similar. The etching threshold fluences were also determined, and were found to be 340,360 and $500 \mathrm{~mJ} / \mathrm{cm}^{2}$ for wet-, dry- and plasma assisted etchings, respectively.

The morphological studies on the etched holes showed that $\mathrm{Ra}$ roughness parameter increased with increasing the fluence for LIBWE and LIPAA, while in the case of the dry etching $\mathrm{Ra}$ was approximately constant and much lower than for the other two methods. However, Ra values normalized by the actual etched depth values were similar. This means that LIBDE method is most suitable for preparation of fine structures displaying small etched depth and roughness, however, when higher depth is required with relatively low roughness then the LIPAA is more suitable at fluences below $1 \mathrm{~J} / \mathrm{cm}^{2}$.

The EDX analysis of the etched hole bottoms demonstrated that significant numbers of tin atoms were built into the surface layer of the fused silica during the procedure. Our calculations proved that the tin content is the highest in the case of wet etching and the lowest for the dry etching. We believe that the developed metal-fused silica mixture layer plays an important role in the etching processes for all of the investigated procedures. This can explain why the LIBDE method is not a real single-pulse method. In Fig.4.c it can be seen that there is some etching even after the $10^{\text {th }}$ laser pulse, too. This can be due to the modified surface layer having relatively large absorption coefficient. The thickness of this layer decreases shot-by-shot, and it operates as a new absorbing layer resulting in further etching until it disappears (Fig.9).

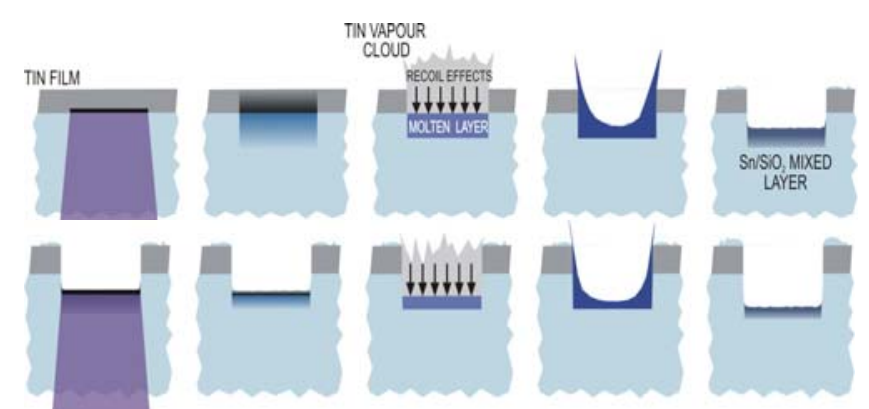

Fig. 9 Main steps of the laser induced backside dry etching process during the $1^{\text {st }}$ (upper row) and the $2^{\text {nd }}$ (lower row) laser pulses

In the cases of LIBWE and LIPAA the etching is a complex process. During the LIBWE the etching mechanism consists of a traditional, expanding bubble-induced etching and additionally, after the first laser pulse an absorbing layer assisted material removal. During the first pulse irradiation several tin atoms built in the fused silica surface resulting in the modified layer on the bottom of the etched hole (Fig.10). At further irradiation the majority of the pulse energy is absorbed in the liquid tin, however, a significant part of the energy is absorbed in the tin-fused silica mixed layer, too (Fig.10).

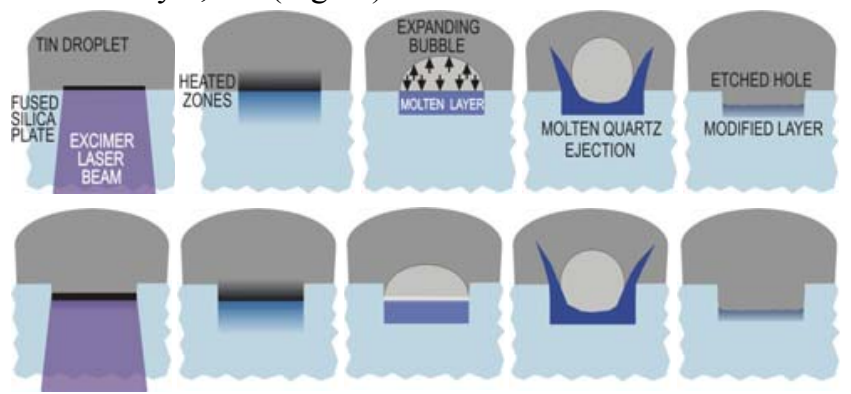

Fig. 10 Main steps of the laser induced backside wet etching process during the $1^{\text {st }}$ (upper row) and the $2^{\text {nd }}$ and forthcoming

(lower row) laser pulses

We suggest, that after each pulse the modified surface layer containing tin atoms is redeveloped influencing the further material removal. A dynamic equilibrium is evolved shot by shot, which is in good agreement with the etch rate - number of pulses dependence (Fig.4.a).

In the case of LIPAA the etching mechanism consists of a traditional plasma assisted ablation, and similarly to the LIBWE, after the first laser pulse an absorbing layer assisted material removal also contributes and leads to the linear pulse dependence corresponding to the Fig.4.b.

During the $1^{\text {st }}$ pulse the excimer laser pulse energy is completely absorbed in the irradiated tin plate resulting in high energetic plasma cloud. This hits against the almost contacting fused silica surface causing etching. During the etching process several tin atoms built into the fused silica surface resulting in the modified layer on the bottom of the etched hole. In consequence of the etching of both the tin and fused silica surfaces the distance between them increases.

For further laser pulses the majority of the pulse energy is absorbed in the tin plate resulting in new plasma ejection, however, a significant part of the laser energy is absorbed in the tin-fused silica mixed layer, too (Fig.11). 


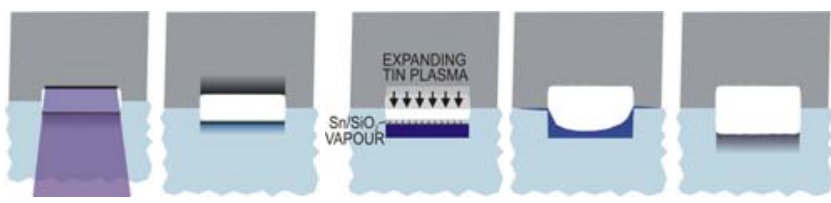

Fig. 11 Main steps of the laser induced plasma assisted ablation process during the $2^{\text {nd }}$ and forthcoming laser pulses

The increased gap would decrease the etch rate however the laser energy absorbed in the modified layer promotes the etching affect of the plasma cloud. After each pulse the modified surface layer is redeveloped influencing the further etching. A dynamic equilibrium is evolved shot by shot, which can explain the good agreement between the calculated thickness of the molten fused silica layer and the measured etch rate, even when there is a small gap between the fused silica and tin plates (Fig.8.c).

\section{Acknowledgements}

The authors gratefully acknowledge the financial support of the Hungarian Scientific Research Foundation OTKA (K 67818, CNK 78549).

\section{References}

[1] H. Niino, Y. Yasui, X. Ding, A. Narazaki, T. Sato, Y. Kawaguchi, A. Yabe: J. Photochem. and Photobio. A: Chemistry, 158, (2003) 179.

[2] R. Böhme, K. Zimmer: Appl. Surf. Sci., 247, (2005) 256.

[3] Cs. Vass, D. Sebők, B. Hopp: Appl. Surf. Sci., 252, (2006) 4768.

[4] R. Böhme, T. Otto, K. Zimmer: Appl. Surf. Sci., 252 (2006) 4392.

[5] R. Böhme, D. Spemann, K. Zimmer: Thin Solid Films, 453/454, (2004) 127.

[6] G. Kopitkovas, T. Lippert, C. David, A. Wokaun, J. Gobrecht: Thin Solid Films, 453/454, (2004) 31.

[7] R. Böhme, K. Zimmer: Appl. Phys. A 83, (2006) 9.

[8] Cs. Vass, B. Hopp, T. Smausz, F. Ignácz: Thin Solid Films, 453/454, (2004) 121.

[9] K. Zimmer, R. Böhme, D. Ruthe, B. Rauschenbach: Appl. Phys. A 84, (2006) 455.
[10] K. Zimmer, R. Böhme, D. Hirsch D, B. Rauschenbach: J. Phys. D: Appl. Phys., 39 (2006) 4651. [11] A. A. Tseng: Phys. Stat. Sol. (A), 204 (2007) 709.

[12] B. Hopp, Cs. Vass and T. Smausz: Appl. Surf. Sci., 253 (2007) 7922.

[13] T. Smausz, T. Csizmadia, N. Kresz, C. Vass, Zs. Márton, B. Hopp: Appl. Surf. Sci., 254 (2007) 1091.

[14] B. Hopp, T. Smausz, T. Csizmadia, J. Budai, A. Oszkó and G. Szabó: J. Phys. D: Appl. Phys., 41, (2008) 175501 (6pp)

[15] J. Ihlemann: Appl Phys A 93, (2008) 65.

[16] R. Böhme, K. Zimmer, B. Rauschenbach: Appl. Phys. A 82, (2006) 325.

[17] K. Sugioka, K. Obata, M.H. Hong, D.J. Wu, L.L. Wong, Y.F. Lu, T.C. Chong, K. Midorikawa: Appl. Phys. A 77, (2003) 251.

[18] K. Sugioka, K. Obata, K. Midorikawa, M.H. Hong, D.J. Wu, L.L. Wong, Y.F. Lu, T.C. Chong: J. Photochem. and Photobio. A: Chemistry 158 (2003) 171.

[19] Y. Hanada, K. Sugioka, I. Miyamoto, K. Midorikawa: Appl. Surf. Sci., 248 (2005) 276.

[20] G. Kopitkovas, T. Lippert, N. Murazawa, C. David, A. Wokaun, J. Gobrecht, R. Winfield: Appl. Surf. Sci., 254, (2007) 1073.

[21] S. Pissadakis, R. Böhme, K. Zimmer: Optics Express 15; (2007) 1428.

[22] K. Zimmer, R. Böhme: Opt. and Lasers in Eng., 43, (2005) 1349.

[23] A. Salleo, T. Sands, F.Y. Génin: Appl. Phys. A71, (2000) 601.

[24] J. Zhang, K. Sugioka, K. Midorikawa: Appl. Phys. A 69, (1999) S879.

[25] Cs. Vass, K. Osvay, B. Hopp, Z. Bor: Appl. Phys. A87, (2007) 611.

[26] B. Hopp, Cs. Vass, T. Smausz and Zs. Bor: J. Phys. D: Appl. Phys. 39, (2006) 4843.

[27] J. Goldstein, D.E. Newbury, D.C. Joy, C.E. Lyman, P. Echlin, E. Lifshin, L.C. Sawyer, J.R. Michael: Scanning Electron Microscopy and X-ray Microanalysis; Springer,: 2003-02; ISBN: 0306472929

(Received: December 4, 2009, Accepted: February 15, 2010) 